\title{
The Ventral Midline Thalamus Contributes to Strategy Shifting in a Memory Task Requiring Both Prefrontal Cortical and Hippocampal Functions
}

\author{
Thibault Cholvin, ${ }^{\star}$ Michaël Loureiro, ${ }^{\star}$ Raphaelle Cassel, ${ }^{\star}$ Brigitte Cosquer, Karine Geiger, David De Sa Nogueira, \\ Hélène Raingard, Laura Robelin, Christian Kelche, Anne Pereira de Vasconcelos, and Jean-Christophe Cassel \\ Laboratory of Cognitive and Adaptive Neurosciences, UMR 7364, University of Strasbourg-National Center of Scientific Research (CNRS), Federal Research \\ Institute 37 of Neurosciences, CNRS Research Group 2905, Faculty of Psychology, F-67000 Strasbourg, France
}

Electrophysiological and neuroanatomical evidence for reciprocal connections with the medial prefrontal cortex $(\mathrm{mPFC})$ and the hippocampus make the reuniens and rhomboid (ReRh) thalamic nuclei a putatively major functional link for regulations of corticohippocampal interactions. In a first experiment using a new water escape device for rodents, the double-H maze, we demonstrated in rats that a bilateral muscimol (MSCI) inactivation $(0.70 \mathrm{vs} 0.26$ and $0 \mathrm{nmol})$ of the $\mathrm{mPFC}$ or dorsal hippocampus (dHip) induces major deficits in a strategy shifting/spatial memory retrieval task. By way of comparison, only dHip inactivation impaired recall in a classical spatial memory task in the Morris water maze. In the second experiment, we showed that ReRh inactivation using $0.70 \mathrm{nmol}$ of MSCI, which reduced performance without obliterating memory retrieval in the water maze, produces an as large strategy shifting/memory retrieval deficit as mPFC or dHip inactivation in the double-H maze. Thus, behavioral adaptations to task contingency modifications requiring a shift toward the use of a memory for place might operate in a distributed circuit encompassing the MPFC (as the potential set-shifting structure), the hippocampus (as the spatial memory substrate), and the ventral midline thalamus, and therein the ReRh (as the coordinator of this processing). The results of the current experiments provide a significant extension of our understanding of the involvement of ventral midline thalamic nuclei in cognitive processes: they point to a role of the ReRh in strategy shifting in a memory task requiring cortical and hippocampal functions and further elucidate the functional system underlying behavioral flexibility.

\section{Introduction}

Diencephalic lesions induce cognitive symptoms reminiscent of those of hippocampal amnesia or prefrontal dysfunctions (Van der Werf et al., 2003; Aggleton et al., 2011; Carlesimo et al., 2011). Experiments in animals suggest that damage to intralaminar and/or anterior thalamic nuclei take part in such effects (Aggleton and Brown, 1999; Gold and Squire, 2006) and that intralaminar or ventral midline thalamic nuclei contribute to spatial memory persistence (Lopez et al., 2009; Loureiro et al., 2012). Anatomical and electrophysiological evidence points to a role of the reuniens $(\mathrm{Re})$ and rhomboid $(\mathrm{Rh})$ nuclei in the information flow between the medial prefrontal cortex (mPFC) and the hippocampus (Vertes, 2006; Vertes et al., 2007; Hoover and Vertes,

\footnotetext{
Received Feb. 20, 2013; revised April 4, 2013; accepted April 6, 2013.

Author contributions: A.P.d.V. and J.-C.C. designed research; T.C., M.L., R.C., B.C., K.G., D.D.S.N., H.R., L.R., and J.-C.C. performed research; C.K. contributed unpublished reagents/analytic tools; T.C., M.L., A.P.d.V., and J.-C.C. analyzed data; A.P.d.V. and J.-C.C. wrote the paper.

This work was supported by the University of Strasbourg, National Center of Scientific Research, Neurex, and National Institute of Health and Medical Research. We are grateful to 0. Bildstein, D. Egesi, and G. Edomwonyi for their assistance in animal care.

*T.C., M.L., and R.C. contributed equally to this work.

The authors declare no competing financial interests.

Correspondence should be addressed to Dr. Jean-Christophe Cassel, Laboratory of Cognitive and Adaptive Neurosciences, UMR 7364, University of Strasbourg-National Center of Scientific Research, 12 rue Goethe, F-67000 Strasbourg, France. E-mail: jcassel@unistra.fr.

DOI:10.1523/JNEUROSCI.0771-13.2013

Copyright $@ 2013$ the authors $\quad 0270-6474 / 13 / 338772-12 \$ 15.00 / 0$
}

2012). Although the Re projects to the mPFC, ventral CA1, and subiculum, the Rh has additional connections with other cortices, ventral striatum, and amygdala (Su and Bentivoglio, 1990; Dolleman-Van der Weel and Witter, 1996; Vertes et al., 2006; Xu and Südhof, 2013). Recently, Hoover and Vertes (2012) described Re neurons projecting to both structures via axon collaterals, placing the Re at a hub position between the MPFC and the hippocampus. Hembrook and colleagues (Hembrook and Mair, 2011; Hembrook et al., 2012) demonstrated a specific involvement of ReRh in cognitive tasks requiring interactions between the two aforementioned structures but not in those requiring only one of them. Xu and Südhof (2013) found a role of the Re in fear memory generalization. We recently found that reversible inactivation of the ReRh had no influence on recent memory retrieval in the water maze, a typical hippocampus-dependent task (Loureiro et al., 2012). The mPFC has a crucial role in executive functions, behavioral flexibility, and strategy shifting (Ragozzino et al., 2003; Floresco et al., 2008). We therefore hypothesized that, in a task requiring both memory for location and strategy selection/shifting, and thus cortico-hippocampal contributions, rats subjected to inactivation of the ReRh might be impaired but retain the capacity to perform on a typical spatial navigation task. Using a novel testing device, the double-H maze (Pol-Bodetto et al., 2011; Cassel et al., 2012), we designed a training protocol in which rats used two learned escape response sequences (right-left or left-left turns) according to where they 
were released in the maze. Subsequently, in a misleading probe trial, we tested their ability to shift from a previously useful but now inefficient response to a strategy based on memory for place. A first experiment evaluated, in the double- $\mathrm{H}$ maze, the sensitivity of our task protocol to MSCI inactivation of the MPFC or dorsal hippocampus (dHip). A second experiment assessed the effects of ReRh inactivation. Before the double-H maze task, all rats were trained drug-free in a classical Morris water-maze task and given a probe trial after inactivation of the dHip or mPFC (first experiment) or ReRh (second experiment). We show that, whereas only dHip inactivation disrupted memory recall in the water maze, inactivation of the mPFC, dHip, or ReRh disrupted performance in the double- $\mathrm{H}$ maze in relatively comparable ways.

\section{Materials and Methods}

\section{Subjects}

The study adhered to the regulations specified by the European Committee Council Directive of November 24, 1986 (86/609/EEC) and the French Department of Agriculture (references for personal authorization licenses are 67-7 to A.P.d.V., 67-217 to C.K., 67-358 to K.G., and 67-215 to J.-C.C.; the other authors are under the formers' responsibility). The two experiments used a total of 103 male Long-Evans rats aged 3 months at their arrival at the laboratory $(250-300 \mathrm{~g})$. Animals were housed individually in quiet facilities under a $12 \mathrm{~h}$ light/dark cycle (lights on at 7:00 A.M.) with access to food and water ad libitum, controlled temperature, and a hygrometry of $\sim 55 \%$. Before any experiment (surgery or maze training), rats were individually handled for 2 min each day over 5 consecutive days in their housing room. All rats were maintained in this room for the duration of the experiment. For the behavioral evaluations, the rats were brought to the testing room $\sim 1$ min before the start of the first training trial on each day. An identical interval was used for all probe trials (see below).

\section{Surgical procedure}

Rats were anesthetized with a mixture of ketamine $(98 \mathrm{mg} / \mathrm{kg})-$ xylazine $(13 \mathrm{mg} / \mathrm{kg})$ administered intraperitoneally. A stainless steel guide cannula $(0.4 \mathrm{~mm}$ in diameter) was implanted bilaterally in the mPFC or hippocampus and unilaterally in the ReRh. Coordinates were as follows: $+2.7 \mathrm{~mm}$ anteroposterior (AP), $\pm 0.7 \mathrm{~mm}$ mediolateral (ML), and -3.2 $\mathrm{mm}$ dorsoventral (DV) for the $\mathrm{mPFC} ;-3.8 \mathrm{~mm} \mathrm{AP}, \pm 2.4 \mathrm{~mm} \mathrm{ML}$, and $-2.2 \mathrm{~mm}$ DV for the dHip; and $-2.3 \mathrm{~mm} \mathrm{AP}, \pm 1.7 \mathrm{~mm} \mathrm{ML}$, and -6.2 $\mathrm{mm}$ DV from skull, with a mediolateral angle of $15^{\circ}$, for the ReRh. All coordinates are in accordance with Paxinos and Watson (1998). Each guide cannula was secured to the skull by acrylic dental cement and stainless steel screws. At the end of surgery, a sterile stainless steel mandrel $(0.28 \mathrm{~mm}$ in diameter $)$ was inserted into each guide cannula. After the operation, all rats were allowed to recover under a warm lamp for 20-30 min before being placed back into their home cage. They were given a 1 week rest period before the start of behavioral evaluations.

\section{The water-maze task}

The specifications of the water maze and the testing procedures have been described previously (Lopez et al., 2012). In brief, the first pretraining day consisted in one four-trial session using a visible platform $(11 \mathrm{~cm}$ diameter, painted black, protruding $1 \mathrm{~cm}$ above the water surface) located in the southeast quadrant of the pool, starting randomly from each of the four cardinal points at the edge of the pool. A blue curtain surrounded the pool to prevent the use of distal cues. After this first day, the curtain was removed. The rats were then given four successive acquisition trials per day ( $10-15 \mathrm{~s}$ intertrial interval) of a maximum duration of $60 \mathrm{~s}$, starting randomly from each of the four cardinal points from the edge of the pool, to learn the location of a platform $(11 \mathrm{~cm}$ diameter $)$ hidden $1 \mathrm{~cm}$ below the water surface in the northwest quadrant. Training extended over 8 consecutive days. The distance traveled and latency before reaching the platform, the swim speed, and the thigmotactic behavior were recorded and subsequently computed by a video-tracking system (SMART; PanLab). At the end of the acquisition, rats were ran- domly allocated to the group to be subjected to inactivation by MSCI or to be used as control after a PBS infusion in the mPFC, dHip, or ReRh. For the probe trial, no platform was placed in the pool. Thirty minutes after the infusion (see below for the drug infusion protocol), rats were released from the northeast start point and allowed a $60 \mathrm{~s} \mathrm{swim} \mathrm{time} \mathrm{in}$ the pool. We recorded the time spent in each target quadrant and the number of crossings over the target area, which was used as an index of memory precision (Lopez et al., 2012). The annulus is defined as a surface covering the size of the top of the platform enlarged by a 10 -cm-wide annulus. The swim speed and thigmotaxis were also recorded during the probe trial.

\section{The double-H maze task}

Testing apparatus. The apparatus has been conceived, constructed, and validated intra muros. It was located in a $3.64 \times 6.52 \mathrm{~m}$ large room as illustrated in Figure $1 a$. In a top view, the general layout of the apparatus approximately corresponds to the shape of two contiguous Hs (Fig. 1a). The double-H maze is made of three parallel run arms, $160 \mathrm{~cm}$ long $\times 20$ $\mathrm{cm}$ wide, connected to each other at the level of their center by a $160 \mathrm{~cm}$ long $\times 20 \mathrm{~cm}$ wide central corridor (Fig. 1a); a photograph of the device can be found in the study by Pol-Bodetto et al. (2011). Each of these arms is delimited by transparent side walls, $35 \mathrm{~cm}$ high. By convention, the two opposing arms in the middle are designed as north (N) and south (S), respectively. The extremities of both other pairs of side arms correspond to our four potential target locations. They are termed northwest (NW), northeast (NE), southwest (SW), and southeast (SE) hereafter (Fig. 1a). All elements of the apparatus are made of transparent Plexiglas. The double- $\mathrm{H}$ device was placed on a table, $80 \mathrm{~cm}$ from the floor, in a large room with several well-contrasted cues on the walls (black disk, black triangles, orange-painted heating ducts). Additional details concerning this test and its possible applications can be found in the studies by Pol-Bodetto et al. (2011), Schumacher et al. (2011), Cassel et al. (2012), Lecourtier et al. (2012), and Kirch et al. (2013).

Training protocol. The double-H was filled with an approximate volume of $170 \mathrm{~L}$ of water $\left(21^{\circ} \mathrm{C}\right)$ to a height of $15 \mathrm{~cm}$. The water was made opaque by addition of powdered milk $(\sim 1.5 \mathrm{~g} / \mathrm{L})$. A platform, $11 \mathrm{~cm}$ diameter $\times 14 \mathrm{~cm}$ high, was immersed $1 \mathrm{~cm}$ beneath the water surface at the extremity of the NE arm (for details, see Fig. 1b). For each rat, the task consisted of learning to swim from the start point-which was changed randomly between $\mathrm{S}$ and $\mathrm{N}$ - to the escape platform, which was always located at the extremity of the NE arm. A first day of pretraining habituated the rats to the testing device and the water. For only this pretraining session, the water was left without milk, the platform protruded $1 \mathrm{~cm}$ above the water surface, and the rats were given four consecutive trials, for which the platform was located at the end of the SW arm and the rats were released from the NW. A transparent guillotine door blocked the access to the central corridor, so that rats could only swim straight to the opposite arm. Over the 4 following training days, the rats were also given four daily trials, but now the water was opaque and the platform was immersed at the extremity of the NE arm. These trials were separated from each other by a $10 \mathrm{~s}$ interval. The rats were released in the maze either from the $\mathrm{N}$ or the $\mathrm{S}$ arm in a randomized order (e.g., S, N, S, N on day $1 ; \mathrm{S}, \mathrm{N}, \mathrm{N}, \mathrm{S}$ on day $2 ; \mathrm{N}, \mathrm{S}, \mathrm{N}, \mathrm{S}$ on day 3 , and N, S, N, S on day 4). When released from the $S$, the rats had to turn once right and then left to reach the platform directly. When released from the $\mathrm{N}$, the correct sequence consisted of two consecutive left turns. When the rats were released from the $\mathrm{N}$, the $\mathrm{S}$ arm was closed by a transparent guillotine door to prevent any entry and vice versa. Each trial, whatever the protocol, lasted for a maximum of $60 \mathrm{~s}$. When the rat did not reach the platform within this delay, it was gently guided to the platform by the experimenter. Once a rat had climbed on the platform, it was left there for $10 \mathrm{~s}$ before the next trial was started. The variables recorded were the distance and latency to reach the target arm as well as the swim velocity.

Probe trial. Two probe trials were given: (1) one $24 \mathrm{~h}$ after the first $2 \mathrm{~d}$ of training and (2) another one $24 \mathrm{~h}$ after 2 additional days of training (Fig. 1c). For both probe trials, the platform was removed from the water maze. The probe trial duration was $60 \mathrm{~s}$. All rats were released from the SW arm, with the entry of NW arm closed by a guillotine door. This procedure (1) prevented a repeat of the behavior displayed during maze 


\section{a Double-H in testing room}

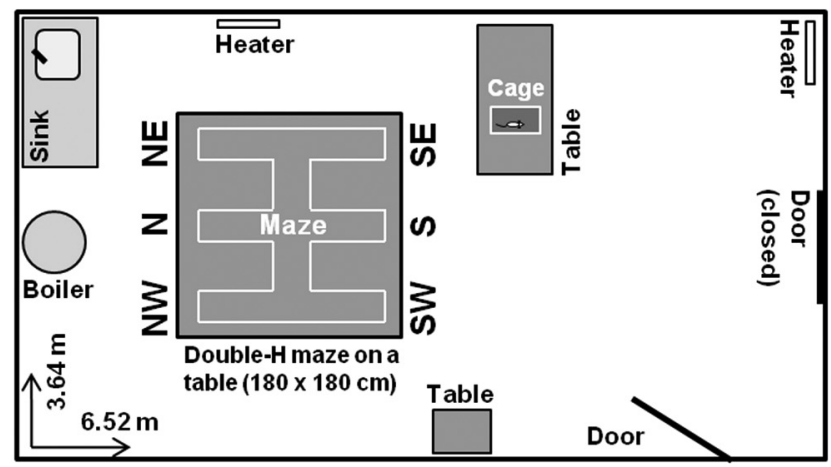

\section{b Training configurations}
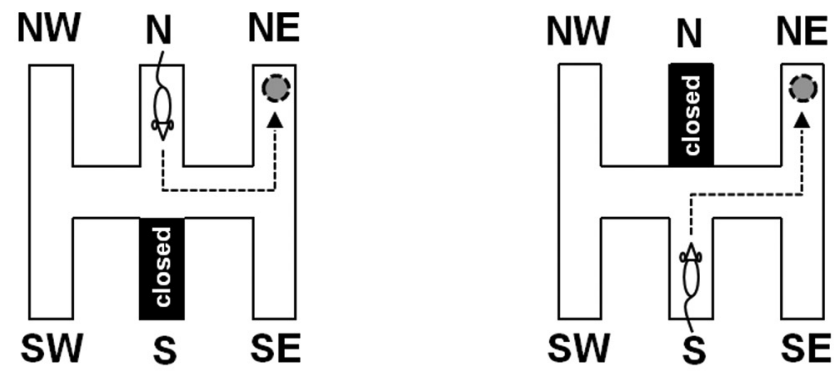

C Time line (probe trials)

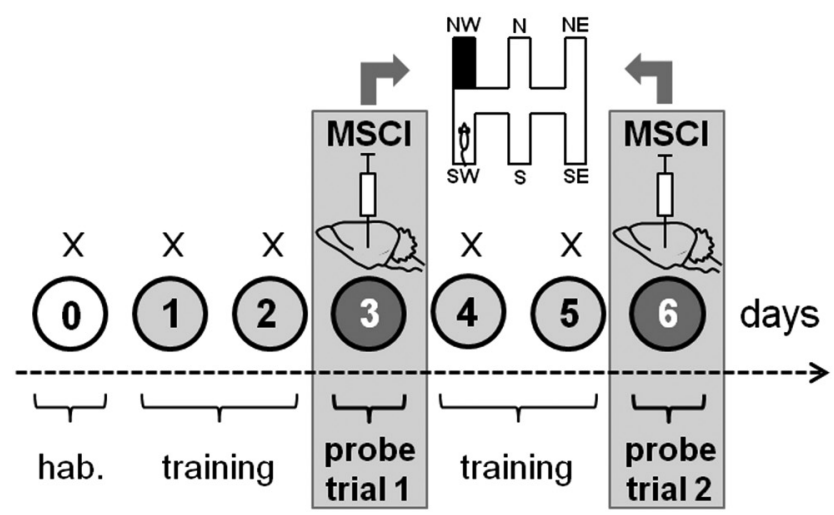

Figure 1. The double-H test, training, and reversible inactivation protocols. $\boldsymbol{a}$, Top-view drawing of the overall organization of the room in which the double-H device was installed (Maze). For a photograph of the device, see the study by Pol-Bodetto et al. (2011). b , Illustration of the configurations used during maze training. The rats were given four daily trials for which they were released twice from the S and twice from the N (in a randomized order, e.g., N, S, S, N on day 1, S, N, S, N on day 2, etc.). The platform was always located in the NE arm. The arm opposite to the one in which the rats were placed at the start of each of the four daily trials was always closed by a transparent guillotine door (it corresponds to the black-colored arm). The most direct swim paths to the escape platform consisted of successive left-left or right-left turns. This training procedure did not prevent spatial learning because rats do shift from a response memory-based to a spatial-memory-based strategy during negative feedback in a misleading probe trial (Cassel et al., 2012).c, Time line of the training days and probe trials in the double- $\mathrm{H}$ test. After a habituation day ( 0 ), rats were trained for 2 consecutive days $(1,2)$, given a first $24 \mathrm{~h}$ delayed probe trial $(3)$, trained for two additional days $(4,5)$, and given a second $24 \mathrm{~h}$ delayed probe trial (6). Thirty minutes before each probe trial, approximately half of the rats were infused with 0.26 or $0.70 \mathrm{nmol}$ in $1 \mu$ l of MSCl in the MPFC or dHip in the first experiment or with 0.26 or $0.70 \mathrm{nmol}$ in $0.3 \mu \mathrm{lof} \mathrm{MSCl}$ in the ReRh in the second experiment, with the others being infused with an equivalent volume of PBS as a control. For both probe trials, rats were released from the SW arm, the NW one was closed by a guillotine door, and the platform had been removed from the device. Each probe trial lasted for $60 \mathrm{~s}$. Before being trained and tested in the double-H maze, all rats had been subjected to a classical spatial learning task in the water maze and given $\mathrm{mPFC}$, dHip, or ReRh inactivation before a standard probe trial at a $24 \mathrm{~h}$ post-acquisition delay. habituation (i.e., swim directly from SW to NW), (2) made one of the learned successive turn sequences impossible (i.e., the left-left sequence), (3) did not disable the right-left sequence, and (4) allowed rats to shift from a strategy based on the right-left turn sequence to a spatial approach of the task (search in target arm) and to do so either directly or during negative feedback (corresponding to entering the $\mathrm{N}$ arm after successive right and left turns). It is noteworthy that, because rats were released from the SW arm, which corresponds to a shift of their start place in the device relative to the testing environment by only $60 \mathrm{~cm}$ to the left, the visuospatial context at the start of the probe trial was relatively comparable with that of all training trials started from the $S$ arm. Therefore, the potential of the probe trial to mislead was extremely high. Indeed, in our previous experiments, we found that, after such a weak shift of the start point, $\sim 90 \%$ of the rats first behaved as if they had been released from the usual training arm (Pol-Bodetto et al., 2011; Cassel et al., 2012). For their first swim track, rats thus had the possibility to adopt the right-left turn sequence (leading them to the $\mathrm{N}$ arm) or immediately use a cognitive map, leading them to the arm of the former platform location, namely the NE one. In cases of a right-left turn as their first approach to the task, given the $60 \mathrm{~s}$ duration of the probe trial, they could subsequently correct for a spatial-memory-based search, which is usually manifested by an increased time spent in the arm in which they were used to find the platform during training (Pol-Bodetto et al., 2011; Cassel et al., 2012). Whether the shift to a spatial strategy was immediate or not, evidence for searching the platform at the correct place can be interpreted as the consequence of a strategy shift. The variables recorded and analyzed were the time spent in the former target arm (i.e., NE, termed the place arm hereafter) and the time spent in the arm to which successive right-left turns were leading (i.e., $\mathrm{N}$, termed response arm hereafter).

\section{Functional reversible inactivation by MSCI infusions} In contrast to lidocaine or tetrodotoxin, MSCI reportedly induces a deep inactivation of neurons in its diffusion radius without changing the excitability of the fibers en passant therein (Edeline et al., 2002; van Duuren et al., 2007). As described previously (Lopez et al., 2012; Loureiro et al., 2012), all rats were first habituated to be handled and maintained as for drug infusions. Such handling lasted for 5 consecutive days. For the infusions, rats were gently restrained by hand, the mandrels were removed, and infusion needles $(0.28 \mathrm{~mm}$ diameter $)$ were slowly inserted into the guide cannula with the tip of the needle protruding $1.0 \mathrm{~mm}$ beyond the tip of the guide cannula. The needle was connected to a $10 \mu \mathrm{l}$ Hamilton syringe by polyethylene tubing. Using a micropump (CMA100; CMA/Microdialysis), MSCI (Sigma; dissolved in PBS) was infused over $60 \mathrm{~s}$ to induce inactivation. In $\mathrm{mPFC}$ and dHip, rats received on each site 0.26 or $0.70 \mathrm{nmol}$ of MSCI in $1 \mu \mathrm{l}$ of PBS (thus, $1 \mu \mathrm{l}$ of a 0.03 and 0.08 $\mu \mathrm{g} / \mu \mathrm{l}$ solution, respectively) before the probe trial in the water maze and before each probe trial in the double-H maze. In the ReRh group, they received 0.26 or $0.70 \mathrm{nmol}$ of MSCI in $0.3 \mu \mathrm{l}$ of PBS (thus, $0.3 \mu \mathrm{l}$ of a 0.10 and $0.27 \mu \mathrm{g} / \mu \mathrm{l}$ solution, respectively) before the probe trial in the water maze and before each probe trial in the double-H maze. A smaller PBS volume was used in ReRh rats to limit MSCI diffusion, these nuclei being small. All controls $(0 \mathrm{nmol})$ received an infusion of an identical volume of PBS (i.e., $1 \mu \mathrm{l}$ in the mPFC and dHip and $0.3 \mu \mathrm{l}$ in the ReRh). After the infusion, the needle was left in place for an additional $30 \mathrm{~s}$ before being slowly retracted. All infusions were performed in the room in which the rats were maintained for the duration of the experiment. Right after the infusion, the rats were returned to their home cage until the start of the probe trial, which occurred $30 \mathrm{~min}$ after infusion in both the Morris water maze and the double-H maze.

\section{Histological verifications}

Ten minutes after an infusion of fluorescent MSCI (Invitrogen), using amounts identical to those used for the experiments, the rats were killed by injection of an overdose of pentobarbital $(200 \mathrm{mg} / \mathrm{kg}$, i.p.) and transcardially perfused with a $4 \%$ phosphate-buffered $(0.1 \mathrm{M})$ paraformaldehyde solution $\left(4^{\circ} \mathrm{C}\right)$. Brains were removed, postfixed for $2 \mathrm{~h}$ in the same fixative at $4^{\circ} \mathrm{C}$, and transferred to a $20 \%$ phosphate-buffered $(0.1 \mathrm{M})$ sucrose solution for $48 \mathrm{~h}$ at $+4^{\circ} \mathrm{C}$ before being snap frozen (isopentane, 
$-40^{\circ} \mathrm{C}$ ) and stored at $-80^{\circ} \mathrm{C}$. Coronal sections $(50 \mu \mathrm{m})$ were cut using a cryostat (Frigocut 2800; Reichert Jung) in serial sections, which, in the respective regions of interest defined according to Paxinos and Watson (1998), were collected on gelatin-coated slides and either processed for cresyl violet staining to be viewed under a light microscope or directly observed under a fluorescence microscope to visualize fluorescent MSCI. Fluorescent MSCI produces a red color when excited at $544 \mathrm{~nm}$ under a fluorescence microscope, enabling the detection of the intraparenchymal presence, location, and diffusion radius of the drug. This fluorescent MSCI was shown previously to have similar electrophysiological and behavioral effects as the classical form of MSCI (Allen et al., 2008). It is noteworthy that the molecular weight of fluorescent MSCI is slightly more than five times larger than the weight of the usual nonfluorescent molecule (i.e., $\sim 607$ vs 114 ). This difference may lead to a possible underestimation of the diffusion radius of the non-fluorescent MSCI used to perform the inactivation. Despite this drawback, the fluorescent MSCI has the advantage to enable several verifications, including the following: (1) our infusion devices were still working at the end of our experiments; (2) the presence of the drug could be evidenced in the appropriate target region; and (3) the spreading radius of fluorescence was larger with the largest amount infused than with the smallest one, thereby documenting in situ a "dose" effect. It is also noteworthy that Allen et al. (2008) found that the fluorescent and nonfluorescent MSCI infused into the dorsomedial region of PFC produced relatively comparable effects in a delayed instrumental response task. A final note concerns the possibility that significant amounts of MSCI could have spread to regions adjacent to the regions we wanted to reversibly inactivate, as shown by Edeline et al. (2002). Although such spreading (and thus unspecific effects) cannot be excluded in our current experiments, it is emphasized that even the largest amount of MSCI infused (i.e., $0.08 \mu \mathrm{g}$ ) corresponded to a relatively small dose regarding the doses commonly used in the literature (to give two examples of large doses, $1 \mu \mathrm{g}$ by Blum et al., 2006; $8 \mu \mathrm{g}$ by Churchwell and Kesner, 2011). The spreading of MSCI is also a reason why, conversely to the majority of experiments published so far with druginduced functional inactivation, we have decided to use a dose-effect approach, as done recently by Hembrook et al. (2012), and to infuse relatively small volumes. For this concern, it may be useful to remind that, in the experiment by Hembrook et al. (2012), an infusion of 2.5 $\mathrm{nmol}$ of MSCI (and thus approximately three times our highest amount infused) have been performed in the thalamus at a distance $1.5 \mathrm{~mm}$ dorsal to the ReRh as a control: the authors found this control infusion to have no effect on the behavioral output that was disrupted when the same amount of MSCI was infused into the ReRh. Finally, and perhaps even more convincing, in a recent study, Stratford and Wirtshafter (2013) infused up to $100 \mathrm{ng}$ MSCI into contiguous dorsal thalamic nuclei (i.e., the paraventricular and the mediodorsal nuclei), with the infusion sites being $\sim 500 \mu \mathrm{m}$ from each other; they found opposite and thus sitespecific effects on feeding behavior.

\section{Statistical analyses}

Unless otherwise specified and depending on the experiment, data analyses used a two- or three-way ANOVA, with repeated measures when appropriate. In experiment 1 , for training phases in both mazes, we considered the factors structure (mPFC, dHip), inactivation $(0,0.26$, and $0.70 \mathrm{nmol}$ ), and day and, in the double-H maze, also the factor probe trial (PT1, PT2). In experiment 2, we considered the factors inactivation $(0$, 0.26 , and $0.70 \mathrm{nmol}$ ) and day (water maze) or probe trial (double-H maze). When appropriate, all these analyses were complemented by multiple comparisons using the Newman-Keuls test. The time spent in the target quadrant (water maze) or in the place arm (double-H) during the probe trials was also compared with chance using a one-sample $t$ test. Chance levels were $15 \mathrm{~s}$ in the water maze [(surface of one quadrant/ surface of the pool) $\times 60 \mathrm{~s}$ ] and $8.2 \mathrm{~s}$ in the double- $\mathrm{H}$ [(surface of one arm/accessible surface of the maze $) \times 60 \mathrm{~s}$ ]. Values of $p<0.05$ were considered significant. A $\chi^{2}$ analysis was used to compare categorical variables in the double- $\mathrm{H}$ maze.

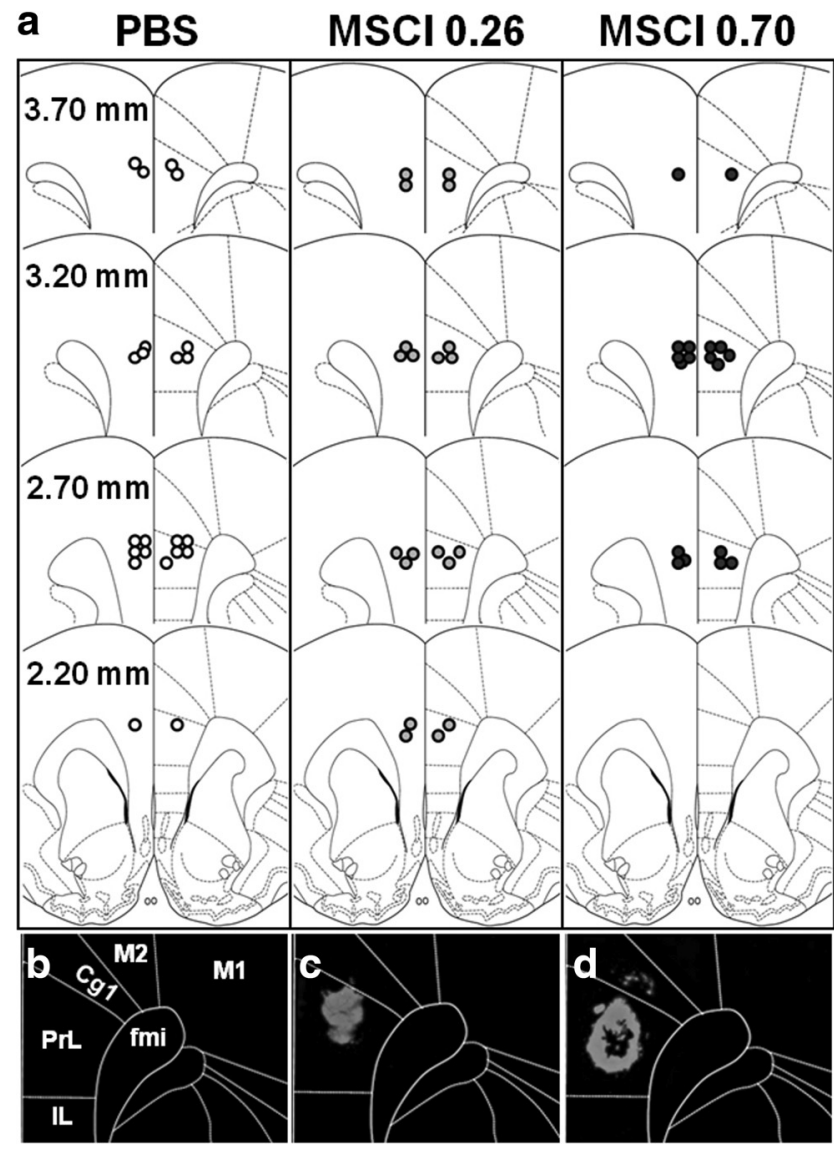

Figure 2. Infusion sites on coronal sections through the MPFC. $\boldsymbol{a}$, Location of the infusion sites in rats subjected to PBS (white circles) or MSCl (gray and black circles, corresponding to 0.26 and $0.70 \mathrm{nmol}$ of $\mathrm{MSCl}$, respectively) infusions before the probe trials. Coordinates are given in millimeters from bregma according to Paxinos and Watson (1998). Each infusion site corresponds to the location of the tip of the infusion needle as identified under a microscope. $c$ and $\boldsymbol{d}$ are photomicrographs showing a representative location/diffusion radius of fluorescent MSCl in the mPFC (bottom; $544 \mathrm{~nm}$ excitation); $\boldsymbol{b}$ is the control (PBS). White lines in $\boldsymbol{b}-\boldsymbol{d}$ delineate the limits of the cingulate cortex, region $1(\mathrm{Cg} 1)$, the prelimbic cortex (PrL), the infralimbic cortex (IL), regions 1 and 2 of the motor cortex (M1,M2), and the forceps minor of the corpus callosum (fmi). Notice the absence of fluorescence after PBS infusion $(\boldsymbol{b})$ and the larger diffusion radius after $0.70 \mathrm{nmol}$ (d) compared with $0.26 \mathrm{nmol}$ (c) fluorescent MSCl infusions; it is likely that the diffusion area of the fluorescent MSCl underestimates the inactivation area obtained with the MSCl used for the behavioral tests.

\section{Results}

\section{Experiment 1}

Location of the infusion sites and diffusion radius of the drug in the $M P F C$ and $d H i p$

Figures $2 a$ and $3 a$ show the location of the injector tips in the mPFC and dHip, respectively, for each group. For mPFC, all injection sites were located in the prelimbic cortex, i.e., between the coordinates 2.2 and $3.7 \mathrm{~mm}$ anterior to bregma. Figure 2, $c$ and $d$, shows typical examples of intraparenchymal diffusion of fluorescent MSCI in the MPFC compared with a PBS infusion (Fig. 2b). Thus, after intra-mPFC infusions, the fluorescent solution remained confined to the $\mathrm{MPFC}$ with maximum fluorescence in the prelimbic cortex and a larger diffusion radius with the largest amount of MSCI (i.e., $0.70 \mathrm{nmol}$ ). For dHip, all injection sites were localized between the coordinates -3.3 and -4.2 $\mathrm{mm}$ posterior to bregma. The extent of the fluorescent MSCI in the dHip is illustrated in Figure 3, $c$ and $d$, compared with PBS (Fig. $3 b$ ). The spread of the solution was confined to the dHip with occasional spread into the overlying cortex. After re- 


\section{a PBS MSCI $0.26 \quad \mathrm{MSCl} 0.70$}

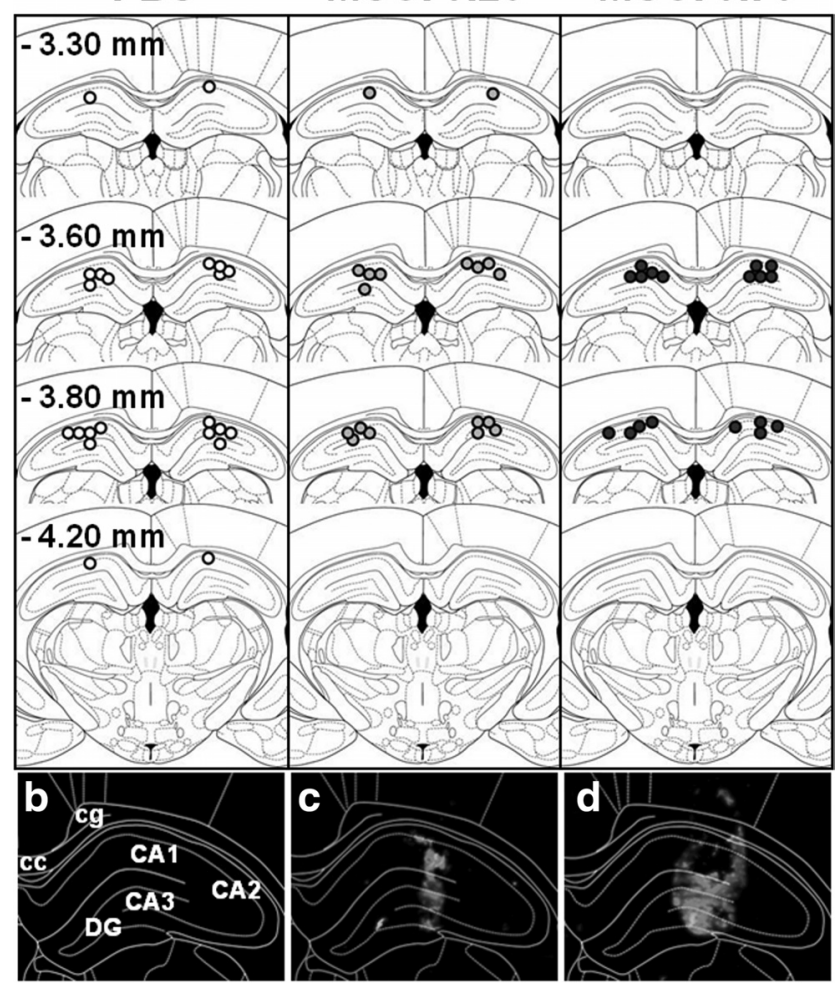

Figure 3. Infusion sites on coronal sections through the dHip. $\boldsymbol{a}$, Location of the infusion sites in rats subjected to $\mathrm{PBS}$ (white circles) or MSCI (gray and black circles, corresponding to 0.26 and $0.70 \mathrm{nmol}$ of $\mathrm{MSCl}$, respectively) infusions before the probe trials. Coordinates are given in millimeters from bregma according to Paxinos and Watson (1998). Each infusion site corresponds to the location of the tip of the infusion needle. $\boldsymbol{c}$ and $\boldsymbol{d}$ are photomicrographs showing a representative location/diffusion of fluorescent MSCl in the $\mathrm{dHip}$ (bottom; $544 \mathrm{~nm}$ excitation); $\boldsymbol{b}$ is the control (PBS). White lines in $\boldsymbol{b}$ - $\boldsymbol{d}$ delineate the limits of the corpus callosum (cc), the cingulum $(\mathrm{cg})$, regions $C A 1, C A 2$, and CA3 of the Ammon's horn, and the gyrus dentatus (DG). Notice the absence of fluorescence after PBS infusion $(\boldsymbol{b})$ and the larger diffusion radius after $0.70 \mathrm{nmol}$ (d) compared with $0.26 \mathrm{nmol}$ (c) MSCl infusions; it is likely that the diffusion area of the fluorescent MSCl underestimates the inactivation area obtained with the MSCl used for the behavioral tests.

moval of seven rats showing misplacement of at least one of the two injection sites, group sizes were as follows: $n_{\mathrm{mPFC}-\mathrm{PBS}}=11$, $n_{\mathrm{mPFC}-0.26 \mathrm{nmol} \mathrm{MSCI}}=10, n_{\mathrm{mPFC}-0.70 \mathrm{nmol} \mathrm{MSCI}}=9, n_{\mathrm{dHip}-\mathrm{PBS}}=11$, $n_{\mathrm{dHip}-0.26 \mathrm{nmol} \mathrm{MSCI}}=9$, and $n_{\mathrm{dHip}-0.70 \mathrm{nmol} \mathrm{MSCI}}=9$.

MSCI inactivation of the dHip, not of the MPFC, impaired retrieval of the platform location in a Morris water maze after drug-free learning

In this experiment, all rats had been equipped with infusion devices before any behavioral training. Starting 1 week after surgery, they were given four daily trials over 8 consecutive days to learn the location of an escape platform hidden in a water maze. After a $1 \mathrm{~d}$ post-acquisition rest, they were tested in a probe trial. During training, the distance to reach the platform declined over days $\left(F_{(7,371)}=392.2, p<0.0001\right)$, showing progressive learning of the platform location (Fig. 4a,b). The statistical analysis of the distance traveled by each rat during the acquisition phase revealed no overall effect of structure $\left(F_{(1,53)}=0.9\right.$, n.s. $)$ or inactivation $\left(F_{(2,53)}=0.2\right.$, n.s. $)$, and there was no significant structure $\times$ inactivation interaction $\left(F_{(2,53)}=1.3\right.$, n.s. $)$. None of the other interactions was significant. The analyses of the latencies, swimming speeds, and thigmotaxis behavior yielded a similar pattern of effects (data not shown). Thus, learning was comparable among the six groups.

ANOVA of the time spent in the target quadrant during the probe trial (Fig. $4 c, d$, left) showed a significant effect of inactivation $\left(F_{(2,53)}=3.4, p<0.05\right)$, structure $\left(F_{(1,53)}=11.0, p<0.01\right)$, and a significant interaction between the two factors $\left(F_{(2,53)}=\right.$ $9.9, p<0.001)$. Post hoc comparisons showed that only rats given $0.70 \mathrm{nmol}$ of MSCI in the dHip were impaired compared with each of the five other groups ( $p<0.001$ in all cases). Comparison of performance in each group with chance level further supported the presence of a deficit in only $\mathrm{dHip}$ rats given the largest amount of MSCI. Indeed, performance significantly exceeded chance in all groups $(p<0.001$ in all cases), except in the dHip$0.70 \mathrm{nmol}$ MSCI group $(p=0.9)$. Analysis of the number of annulus crossings confirmed a performance deficit restricted to dHip-0.70 nmol MSCI rats (Fig. $4 c, d$, right), because post hoc comparisons showed that only these rats were impaired compared with their PBS-infused controls $(p<0.001)$, as well as with each of the other four groups. During the probe trial, thigmotaxis and swim speed were not significantly affected by the inactivation (data not shown; for illustrations of typical swim paths in the different groups, see Fig. $4 e, f)$.

Thus, after drug-free learning of the platform location in the Morris water maze, which is considered a typical hippocampusdependent task, the capability to retrieve a spatial memory was altered by the inactivation of the dHip but only with the higher of the two amounts of MSCI. When infused in the MPFC, the same amount of MSCI did not impair retrieval.

\section{MSCI inactivation of the dHip or the MPFC both disrupted} performance in the double-H maze probe trial after drug-free learning

One week after the probe trial in the water maze, we started drug-free training in the double-H maze. This training was organized over two 2-d periods, with each ending up with a probe trial after a $1 \mathrm{~d}$ rest (Fig. $1 c$ ). Before each probe trial, rats were subjected to MSCI $(0.26$ or $0.70 \mathrm{nmol})$ or PBS infusions into the mPFC or dHip. During training, the distances swum before reaching the platform shortened over days $\left(F_{(3,159)}=59.3, p<\right.$ 0.0001 ), indicating learning (Fig. $5 a, b$ ). There was no significant difference among the six groups, as attested by the absence of significant structure $\left(F_{(1,53)}=0.2\right.$, n.s. $)$, inactivation $\left(F_{(3,53)}=\right.$ 0.3 , n.s. $)$, or structure $X$ inactivation interaction $\left(F_{(2,53)}=0.8\right.$, n.s.) effects. Analyses of the latencies and swim velocities showed comparable significance levels in all groups (data not shown).

The very first two-movement swim sequence in the first probe trial consisted in a succession of right and left turns (leading rats to the $\mathrm{N}$ arm) in $76 \%$ of rats, $14 \%$ swam directly to the former location of the platform (i.e., NE arm), and the last $10 \%$ of animals reached another arm, with no significant difference among groups ( $\chi^{2}$ statistic, n.s.). For the second probe trial, $78 \%$ of rats chose the $\mathrm{N}$ arm, 15\% directly swam to the former platform location (i.e., to the NE arm), and the other rats swam to the SE or $\mathrm{S}$ arms. This behavioral pattern did not differ significantly among mPFC-PBS, mPFC-MSCI, dHip-PBS, and dHip-MSCI groups, regardless of the probe trial and amount of MSCI infused $\left(\chi^{2}\right.$ statistic, n.s.). However, in terms of relative amount of time the various groups spent in the $\mathrm{NE}$ and $\mathrm{N}$ arms, clear-cut differences emerged during probe trials, especially during the second one (Fig. $5 c-g$ ). The $2 \times 2 \times 2$ ANOVA of the time spent in the place $\operatorname{arm}(\mathrm{NE})$ showed significant structure $\left(F_{(1,53)}=11.2, p<0.01\right)$, inactivation $\left(F_{(2,53)}=17.4, p<0.001\right)$, and probe trial $\left(F_{(1,53)}=\right.$ 9.0, $p<0.01)$ effects. None of the interactions was significant. 
a
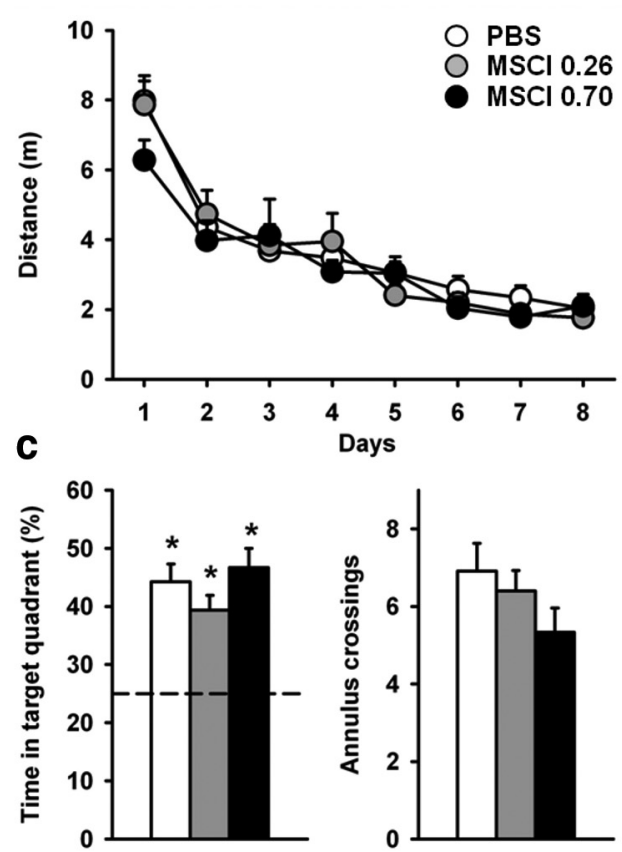

e

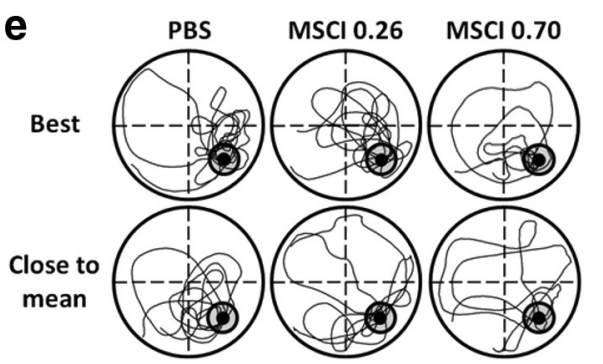

b

dHip
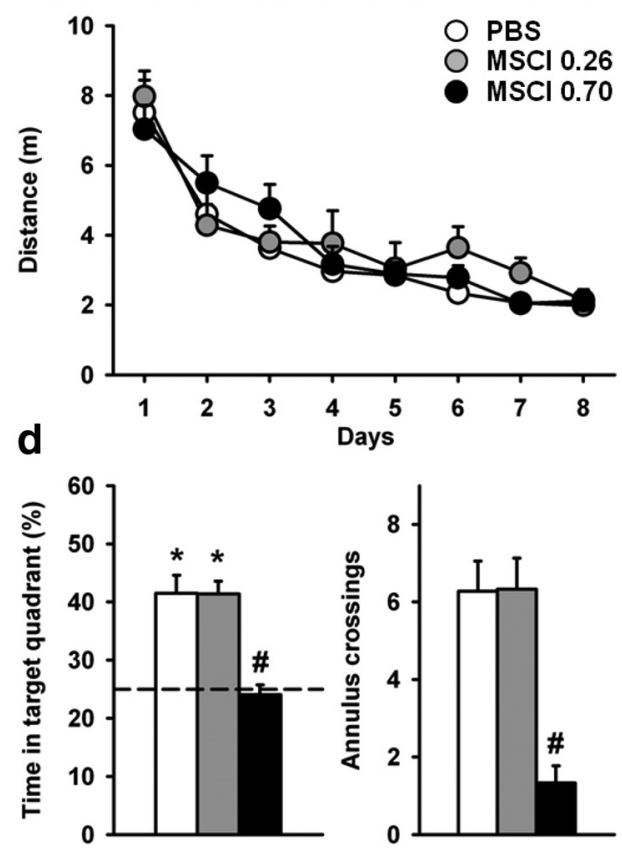

f

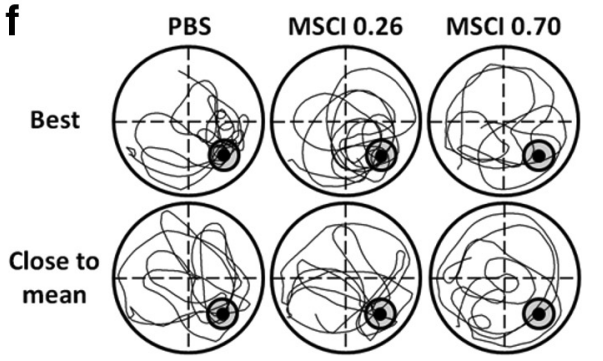

Figure 4. Acquisition curves and effects of dHip or mPFC MSCl inactivation on spatial memory retrieval in the hidden version of the water-maze task. $\boldsymbol{a}, \boldsymbol{b}$, Drug-free acquisition expressed as average \pm SEM distance to reach the platform in the rats that were subsequently tested for a probe trial after bilateral MSCl or PBS infusions into the mPFC or dHip (c-f $)$. All rats were subjected to four daily trials over 8 consecutive days. There was no significant difference among the six groups, in terms of overall performance or learning curves. Mean \pm SEM performance during the probe trial was expressed as percentage time in the target quadrant $(\boldsymbol{c}, \boldsymbol{d}$, left) and number of crossings in the annulus as an index of precision $(\boldsymbol{c}, \boldsymbol{d}$, right). The dashed line in $\boldsymbol{c}$ and $\boldsymbol{d}$ represents chance level (i.e., $25 \%$ ). ${ }^{*} p<0.05$, significant difference compared with chance (i.e., $25 \%$ ); ${ }^{\#} p<0.05$, significant difference compared with control (PBS). All but dHip- $0.70 \mathrm{nmol}$ MSCl rats remembered the platform location and concentrated with comparable precision their searches in the target quadrant where the platform had been located during acquisition. Representative swim paths are shown after mPFC (e) or dHip $(\boldsymbol{f})$ inactivation. The examples are from a rat that showed the best performance, i.e., highest percentage of time spent in the target quadrant (top) and from a rat showing performance closest to the group mean (bottom) in each group. PBS, Infusions of PBS; MSCI 0.26, MSCI 0.70 , infusions of 0.26 and $0.70 \mathrm{nmol}$ of MSCl, respectively.

Multiple comparisons showed that the inactivation with 0.70 nmol of MSCI induced a marked deficit, regardless of region, compared with control rats (Fig. $5 c, d$ ). After PBS infusions, this group was able to search for the platform in the appropriate target, especially during the second probe trial. Indeed, in $\mathrm{MPFC}-$ PBS and dHip-PBS rats, performance was significantly above chance during the second probe trial $(p<0.05)$, as was already the case in the first probe trial for $\mathrm{MPFC}-\mathrm{PBS}$ rats. In $\mathrm{mPFC}$ rats given 0.26 or $0.70 \mathrm{nmol}$ of MSCI, performance did not differ from chance on both probe trials. In dHip rats, for both amounts of MSCI, performance was significantly below chance level on the first probe trial $(p<0.05)$ and did not differ from chance on the second one. The analysis of the time spent in the response $\operatorname{arm}(\mathrm{N}$ arm; Fig. $5 e, f)$ showed no significant effect of the single factors or of any of their interactions, except for the probe trial, during which the overall time was significantly lower on the second compared with the first probe trial $(p<0.05)$ for most groups. Swim tracks from a representative rat of each group are shown in Figure $5 g$.

In summary, rats in the control groups searched for the platform more accurately after $4 \mathrm{~d}$ of training than after only $2 \mathrm{~d}$. The inactivation of the mPFC or dHip disrupted searching in the appropriate arm, with the dHip inactivation effect appearing more pronounced than the mPFC one because the dHip effect was observed for both amounts of MSCI.

\section{Experiment 2}

Location of the infusion sites and diffusion radius of the drug in the ReRh

Almost all infusion sites were found to be localized in the ReRh and most of them between anteroposterior coordinates -1.8 and 2.6 mm from bregma (Fig. $6 a$ ). Figure $6, c$ and $d$, shows a typical example of MSCI diffusion after intra-ReRh infusion of fluorescent MSCI (compare with PBS in Fig. 6b). After removal of six rats, because of misplaced cannula, group sizes were as follows: $n_{\mathrm{ReRh}-\mathrm{PBS}}=11$, $n_{\text {ReRh-0.26 nmol MSCI }}=10$, and $n_{\text {ReRh-0.70 nmol MSCI }}=10$.

MSCI inactivation of the ReRh impaired performance but did not prevent retrieval of the platform location in the Morris

water-maze task after drug-free learning

As in experiment 1, starting 1 week after surgery, the rats were first trained over 8 consecutive days to locate a hidden platform in 
a
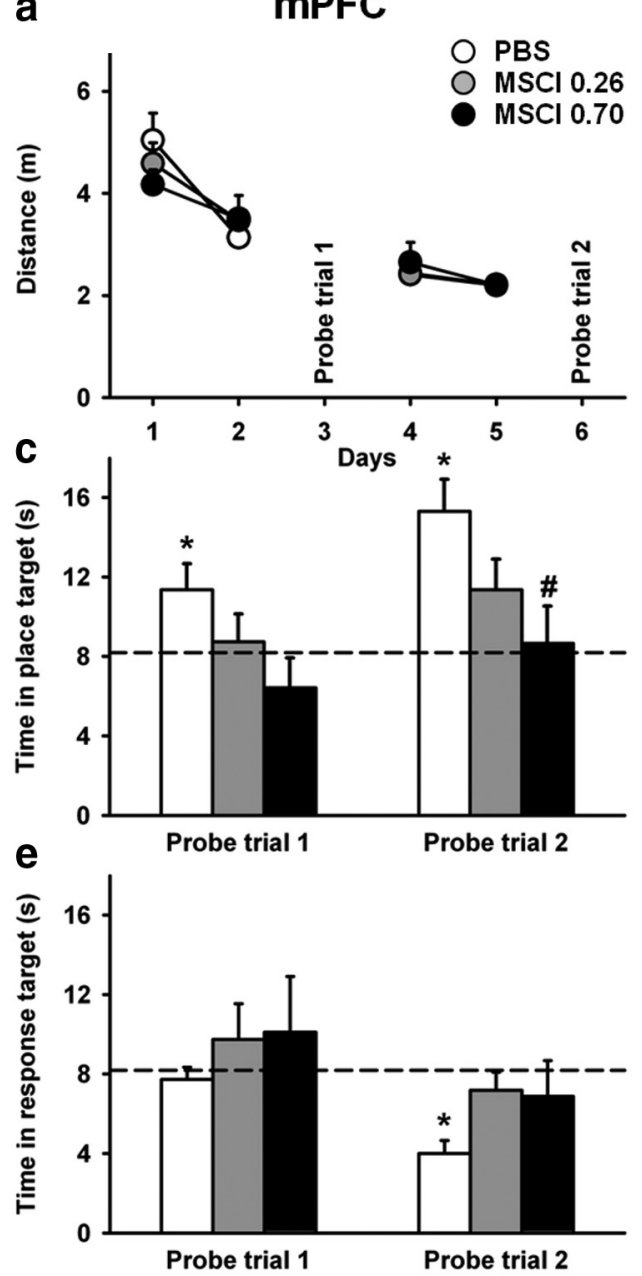

g

PBS

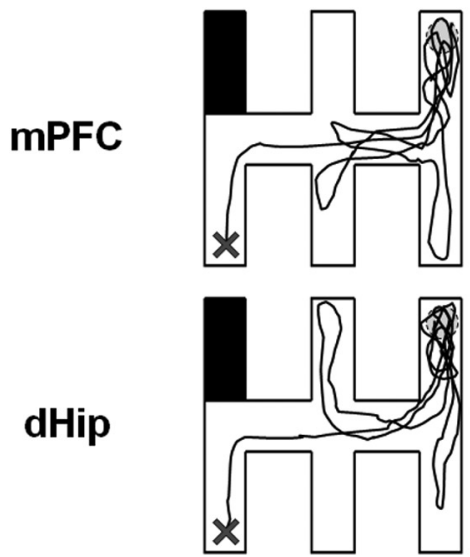

$\mathrm{MSCl} 0.26$
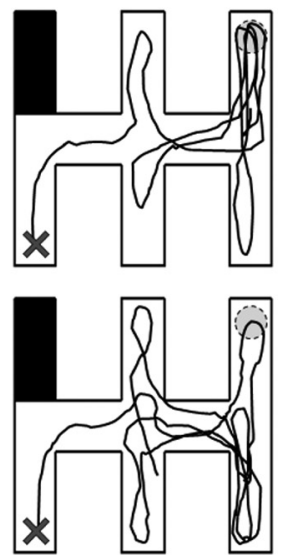

b

dHip

$\mathrm{MSCl} 0.26$
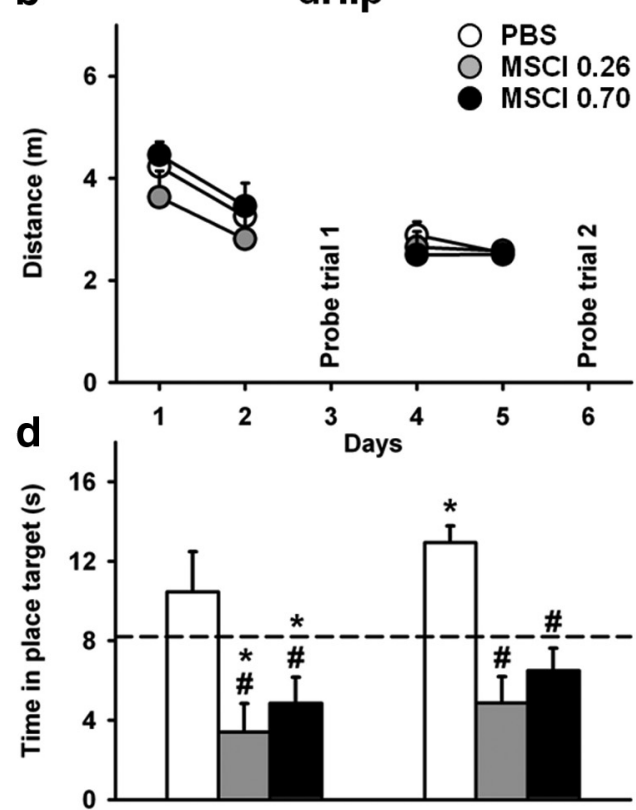

$\mathbf{f}$

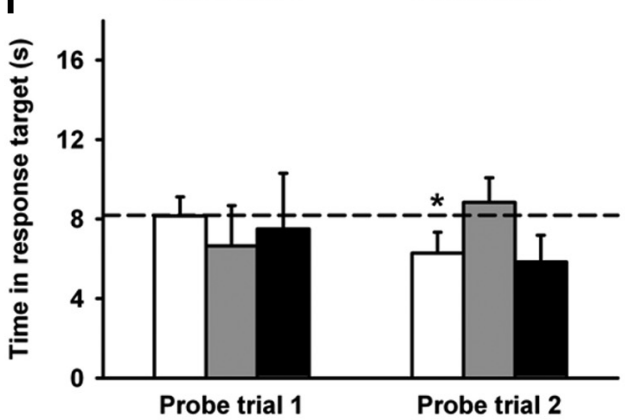

$\mathrm{MSCl} 0.70$

Figure 5. Acquisition curves and effects of dHip or mPFC MSCl inactivation on performance in the double-H maze task. Drug-free acquisition expressed as average \pm SEM distance swum $(\boldsymbol{a}, \boldsymbol{b})$ to reach the platform. All rats were subjected to four daily trials on days $1,2,4$, and 5 . On days 3 and 6 , they were given a probe trial after bilateral infusion of either 0.26 or $0.70 \mathrm{nmol}$ of MSCl or PBS as control. There was no significant difference among the six groups in terms of overall performance or learning curves. Probe trial performance is expressed as the time spent in the place target arm (i.e., $\mathrm{NE} \mathrm{arm}$ ) in cand $\boldsymbol{d}$ and the time spent in the response arm $(\mathrm{N}$ arm $)$ in $\boldsymbol{e}$ and $\boldsymbol{f}$ in rats subjected to infusions into the $\mathrm{mPFC}$ or $\mathrm{dHip}$. Representative swim paths are shown in $\boldsymbol{g}$ for each group; all rats were released from SW arm and taken out of the maze at the end of the $60 \mathrm{~s}$ probe trial. Abbreviations are as in Figure $4 .{ }^{\#} p<0.05$, significant difference compared with PBS; ${ }^{*} p<0.05$, significant difference compared with chance (indicated by the hatched line in $\mathbf{c}-\boldsymbol{f}$ ).

the water maze. They were subsequently tested in a probe trial after a $24 \mathrm{~h}$ post-acquisition rest. During training, the distance to reach the platform declined over days $\left(F_{(7,196)}=50.0, p<\right.$ 0.0001 ), showing progressive learning of the location of the hid- den platform (Fig. 7a). There was no effect of inactivation (PBS vs MSCI, 0.26 and $0.70 \mathrm{nmol} ; F_{(2,28)}=0.3$, n.s.) and no significant inactivation $\times$ day interaction $\left(F_{(14,196)}=1.4\right.$, n.s. $)$. The analyses of the latencies, swimming speed, and thigmotaxis only showed 
a PBS $\mathrm{MSCl} 0.26 \quad \mathrm{MSCl} 0.70$

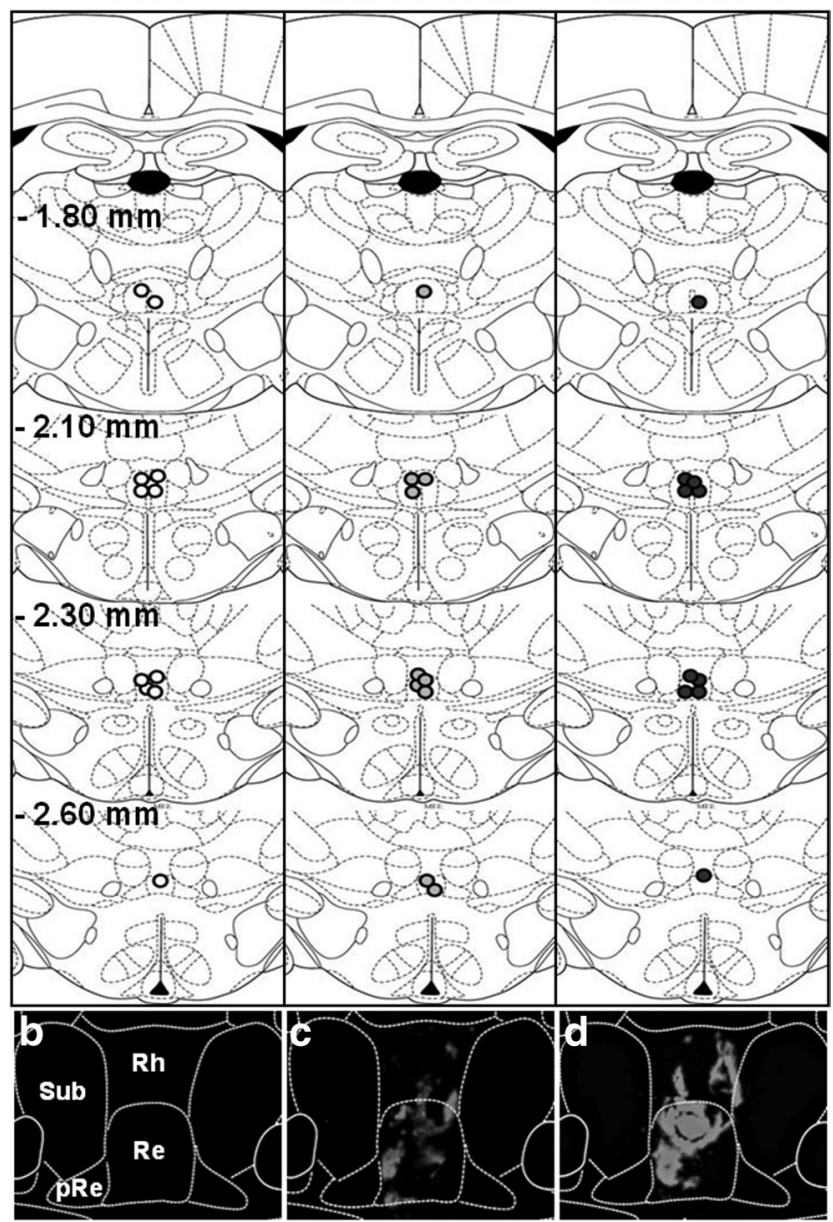

Figure 6. Infusions sites on coronal sections through the midline thalamus. $\boldsymbol{a}$, Site locations were examined in rats subjected to PBS or MSCl infusion before the probe trial. Coordinates are given in millimeters from bregma according to Paxinos and Watson (1998). Each infusion site is represented by white (PBS), gray $(0.26 \mathrm{nmol}$ of MSCl$)$, or dark circles $(0.70 \mathrm{nmol}$ of MSCl) circles, the center of which corresponds to the location of the tip of the infusion needle. $\boldsymbol{c}$ and $\boldsymbol{d}$ are photomicrographs showing the distribution of the fluorescent MSCl solution within the ReRh. The diffusion area appears in red under a fluorescence microscope (excitation $544 \mathrm{~nm}$ excitation); $\boldsymbol{b}$ is the control (PBS). White lines delineate the limits of $R h, R e$, the perireuniens (pRe), and the submedius nucleus (Sub). Notice the absence of fluorescence after PBS infusion ( $\boldsymbol{b}$ ) and the larger diffusion radius after $0.70 \mathrm{nmol}$ (d) compared with $0.26 \mathrm{nmol}$ (c) MSCl infusions; it is likely that the diffusion area of the fluorescent MSCl underestimates the inactivation area obtained with the MSCl used for the behavioral tests.

significant day effects (data not shown). Thus, there was equivalent learning in both groups.

ANOVA of the time spent in the target quadrant during the probe trial (Fig. $7 b$ ) showed a significant effect of inactivation $\left(F_{(2,28)}=5.5, p<0.01\right)$. This effect was attributable to performance that was significantly altered in rats administered 0.26 and $0.70 \mathrm{nmol}$ of MSCI compared with their PBS counterparts ( $p<0.05$ for each comparison). However, these differences did not result in an inability to remember the platform location. Indeed, comparison of performance in each group with chance level showed performance to be significantly above chance in all three groups (PBS, $p<0.001 ; 0.26 \mathrm{nmol}$ of MSCI, $p<0.001 ; 0.70 \mathrm{nmol}$ of MSCI, $p<0.05)$. Although less for both amounts of MSCI compared with PBS ( $p<0.05$; Fig. $7 b)$, it is noteworthy that the number of annulus crossings was much larger than found in dHip- $0.70 \mathrm{nmol} \mathrm{MSCI}$ rats (Fig. $7 c$ a

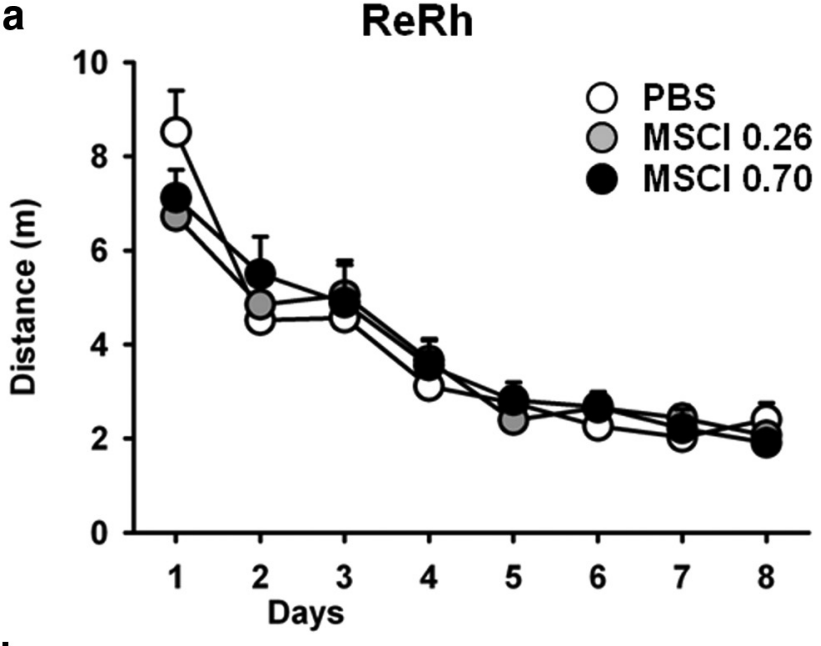

b

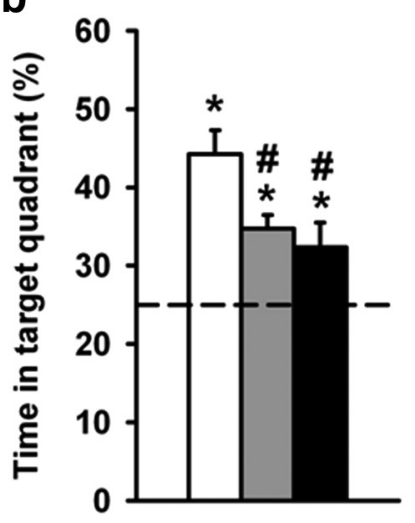

C

d
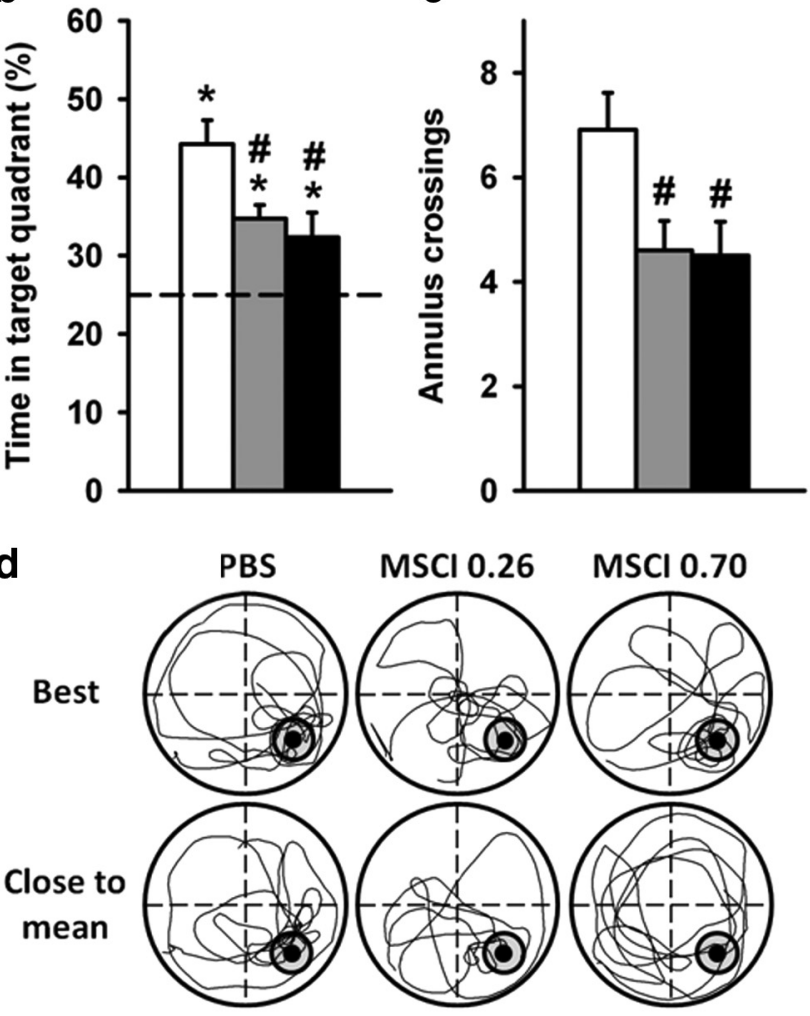

Figure 7. Acquisition curves and effects of ReRh MSCl inactivation on spatial memory retrieval in the hidden version of the water-maze task. $\boldsymbol{a}$, Drug-free acquisition was expressed as average \pm SEM distance to reach the platform in the rats that were subsequently tested for a probe trial after MSCl or PBS infusion. All rats were subjected to four daily trials over 8 consecutive days. There were no significant differences between the three groups. Mean \pm SEM performance during the probe trial were expressed as percentage time in the target quadrant (b) and the number ( \pm SEM) of crossings in the annulus (c).The dashed line in $\boldsymbol{b}$ represents chance level (i.e., 25\%). Abbreviations are as in Figure 4. ${ }^{*} p<0.05$, significant difference compared with chance $(25 \%)$; ${ }^{\#} p<0.05$, significant difference compared with control. $\boldsymbol{d}$, Examples of detailed swim paths during the probe trial. Patterns are shown for a rat displaying the best performance, i.e., highest percentage of time spent in the target quadrant (top) and for a rat showing performance closest to the mean (bottom).

compared with Fig. $4 d$ ). Typical swim paths are shown in Figure $7 d$. Thus, after drug-free learning of the platform location in the Morris water maze, inactivation of the ReRh reduced performance without obliterating the capability to retrieve the platform location. 

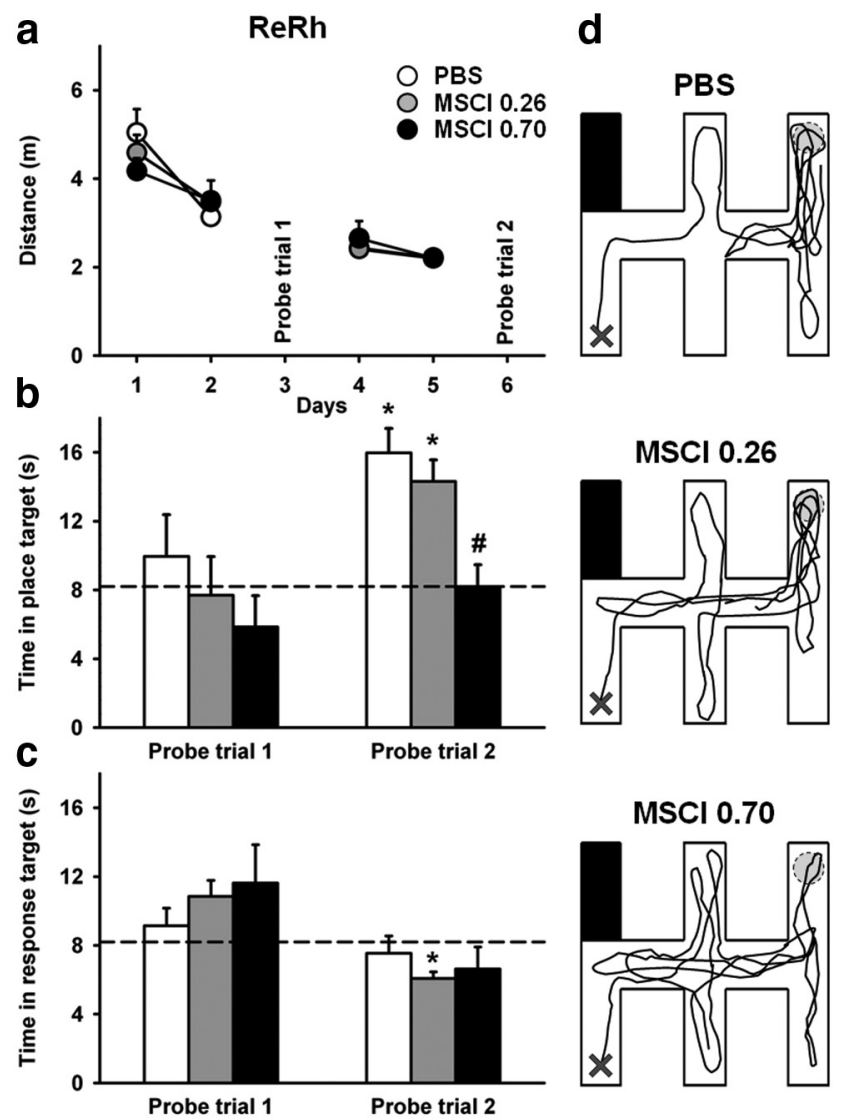

Figure 8. Acquisition curves and effects of ReRh MSCl inactivation on performance in the double-H maze task. Drug-free acquisition was expressed as average \pm SEM distance $(\boldsymbol{a})$ to reach the platform. As in experiment 1, all rats were subjected to four daily trials on days 1, 2,4, and 5 . There were no significant differences between the three groups. On days 3 and 6 , they were given a probe trial after bilateral MSCl or PBS infusion. Probe trial performance was shown as the time spent in the place arm (NE arm) $(\boldsymbol{b})$ and the time spent in the response arm (N arm) (c). Representative swim paths are shown in $\boldsymbol{d}$. The rats were released from the SW arm and taken out of the maze at the end of the $60 \mathrm{~s}$ probe trial. Abbreviations are as in Figure $7 .{ }^{\#} p<$ 0.05 , significant difference compared with $\mathrm{PBS} ;{ }^{*} p<0.05$, significant difference compared with chance (indicated by hatched line in $\boldsymbol{b}$ and $\boldsymbol{c}$ ).

MSCI inactivation of the ReRh disrupted performance in the probe trial of the double-H maze after drug-free learning During training, the distances swum before reaching the platform decreased over days $\left(F_{(3,84)}=86.1, p<0.0001\right)$, indicating learning (Fig. 8a). There was no overall significant difference among the two groups, as attested by the absence of a significant inactivation effect $\left(F_{(2,28)}=1.3\right.$, n.s. $)$. The inactivation $\times$ day interaction was significant $\left(F_{(6,84)}=3.9, p<0.01\right)$ and can be explained by a more pronounced performance improvement in rats from the PBS group between days 1 and 2 compared with both other groups. However, final performance levels (day 5) did not differ among groups. Analysis of latencies yielded similar conclusions, except that the inactivation $\times$ day interaction was not significant $\left(F_{(6,84)}=1.2\right.$, n.s. $)$.

The very first two-movement swim sequence exhibited in the first probe trial consisted of a succession of right and left turns, leading rats to the $\mathrm{N}$ arm in $87 \%$ of rats, whereas none of them swam directly to the former location of the platform, and the other ones reached another arm, with no significant difference among groups ( $\chi^{2}$ statistic, n.s.). For the second probe trial, $83 \%$ of rats exhibited such a behavior (i.e., to go to the $\mathrm{N}$ arm), $10 \%$ swam directly to the former platform location (i.e., to the $\mathrm{NE}$
Table 1. Summary and comparison of $\mathrm{mPFC}$, dHip, and ReRh inactivation effects in the water-maze and double-H-maze tasks (second probe trial)

\begin{tabular}{|c|c|c|c|c|}
\hline \multirow[b]{2}{*}{ Target region } & \multicolumn{2}{|c|}{ Water maze task } & \multicolumn{2}{|c|}{$\begin{array}{l}\text { Double-H task } \\
\text { (2nd probe trial) }\end{array}$} \\
\hline & $0.26 \mathrm{nmol}$ & $0.70 \mathrm{nmol}$ & $0.26 \mathrm{nmol}$ & $0.70 \mathrm{nmol}$ \\
\hline $\mathrm{mPFC}$ & ns & ns & ns & $\downarrow \downarrow \downarrow$ \\
\hline dHip & ns & $\downarrow \downarrow \downarrow$ & $\downarrow \downarrow \downarrow$ & $j$ \\
\hline ReRh & $\downarrow$ & $\downarrow$ & ns & $\downarrow \downarrow \downarrow$ \\
\hline
\end{tabular}

ns, No significant effect; $\downarrow$, performance significantly impaired, memory still there; $\downarrow \downarrow \downarrow$, performance significantly impaired, no evidence of memory.

arm), and the other rats swam to the SE or S arms. This behavioral pattern did not differ significantly among ReRh-PBS and ReRhMSCI groups, regardless of probe trial or amount of MSCI $\left(\chi^{2}\right.$ statistic). However, in terms of relative amount of time the various groups spent in the NE and $\mathrm{N}$ arms, clear-cut differences emerged during the probe trials, especially during the second one (Figs. $8 b-d$ ). The $2 \times 2$ ANOVA of the time spent in the place $\operatorname{arm}(\mathrm{NE})$ showed significant inactivation $\left(F_{(2,28)}=4.5, p<0.05\right)$ and probe trial $\left(F_{(1,28)}=14.9, p<0.001\right)$ effects (Fig. $\left.8 b\right)$. The interaction between both factors was not significant $\left(F_{(2,28)}=1.1\right.$, n.s.). Multiple comparisons showed that $0.70 \mathrm{nmol} \mathrm{MSCI}$ inactivation produced a marked deficit compared with PBS during essentially the second probe trial $(p<0.01)$. In ReRh-PBS rats, performance of the second probe trial was significantly above chance $(p<0.001)$, as was also the case in rats administered 0.26 nmol of MSCI $(p<0.001)$ but not $0.70 \mathrm{nmol}$ of MSCI ( $p=1.0$, n.s.). The analysis of the time spent in the response arm (N; Fig. $8 c)$ showed no significant inactivation $\left(F_{(2,28)}=0.3\right.$, n.s. $)$ or inactivation $\times$ probe trial interaction $\left(F_{(2,28)}=0.7\right.$, n.s. $)$ effects (Fig. 7c). Performance in ReRh- $0.26 \mathrm{nmol} \mathrm{MSCI}$ rats was significantly below chance level. Swim tracks from a representative rat of each group are shown in Figure 8d.

Thus, after 4 learning days, rats from the group with the highest amount of MSCI infused into the ReRh failed to search for the platform as accurately as did the rats of the two other groups during the second probe trial, in line with the performance recorded after the largest MSCI amount in the MPFC or dHip groups (Fig. $5 c, d$ ).

\section{Discussion}

In the Morris water maze, reversible inactivation of the dHip abolished retrieval of a recently acquired spatial memory, an effect not mimicked by mPFC or ReRh inactivation (Table 1). However, in the double- $\mathrm{H}$ maze, the performance disruption produced by $0.70 \mathrm{nmol}$ of MSCI in the ReRh was mimicked by $0.70 \mathrm{nmol}$ of MSCI in mPFC or dHip. Such task/brain structuredependent dissociations suggest that, although the dHip and not the mPFC or ReRh is crucial for recent spatial memory retrieval, all three structures participate in behavioral adaptations invoking a place strategy in the double- $\mathrm{H}$ maze. Indeed, the weak effect of MSCI in the mPFC or ReRh on Morris water-maze performance suggests that the spatial memory system remains operational despite inactivation. ReRh inactivation most probably disrupted the shift-triggering process normally substituting an appropriate place strategy for an inappropriate response one. This cognitive operation might require coordination between the MPFC (as the potential set-shifting structure) and the dHip (as the spatial memory substrate). Given the reciprocal connectivity of the ReRh with both structures, we propose a role for the ReRh in the regulation of strategy shifting underlying behavioral flexibility in adaptation to modifications of environmental demands. 


\section{Hippocampal, not mPFC or ReRh, inactivation disrupts spatial memory retrieval in the water maze}

Regarding $\mathrm{mPFC}$ and dHip inactivation effects on recent memory, our data are in line with the literature (Broadbent et al., 2006; Teixeira et al., 2006; Klur et al., 2009; Lopez et al., 2012). The only exception we know of is the report by Blum et al. (2006) on mPFC inactivation-induced recall deficits. These authors used another task (conditioned fear) and 12 times our highest dose of MSCI, questioning the specificity of the effect. Dolleman-Van der Weel et al. (2009) found that ReRh lesions did not obliterate the ability to learn a water-maze task (confirmed by Loureiro et al., 2012) and to recall the platform location in a probe trial. However, probe trial performance was below that of controls, as found after inactivation in the current study. Based on this weaker performance, the authors proposed a non-mnemonic interpretation suggesting that rats with ReRh lesions showed deficits in strategy shifting. In a previous study (Loureiro et al., 2012), we found normal recent memory retrieval performance, but the inactivation was performed with lidocaine, not with MSCI, and memory was tested $5 \mathrm{~d}$ (not $1 \mathrm{~d}$ ) after acquisition. The fact that ReRh rats showed reduced memory performance in the water maze indicates that MSCI-induced inactivation of the ReRh weakens but does not disrupt hippocampal-dependent cognitive processes contributing to recent spatial memory retrieval.

\section{Inactivation of the mPFC, hippocampus, or ReRh induces deficits in the double-H maze}

The double- $\mathrm{H}$ maze is a novel device that we recently validated using response and place learning tasks, amnestic drugs (PolBodetto et al., 2011), and manipulations of training and testing protocols (Cassel et al., 2012). In one of these protocols, rats were trained to swim from a constant start (S) to a constant target arm (NE). When subsequently tested in a misleading probe trial (as in the present experiments), most rats first engaged a behavior driven by response memory but then shifted to a behavior driven by place memory, indicating a flexibility in their cognitive processing of the situation (Cassel et al., 2012). In the current study, the rats had to use one of two efficient response sequences depending on the arm from which the trials were started. However, for the probe trials, there was no constraint hindering the shift to place memory-driven behavior. Furthermore, all rats were given two probe trials: one after 2 training days and the other after 2 additional days. Interestingly, in all three control groups, the negative feedback-driven shift to place memory-driven behavior eventually occurred in the second probe trial; however, control rats, regardless of infusion site, spent more time in the former target (place) arm than in the others and performed above chance level. This same search pattern was not observed after dHip, mPFC, or ReRh inactivation, suggesting a disruption of either spatial memory processing or strategy shifting. The fact that $\mathrm{dHip}$ rats subjected to MSCI infusions were also impaired in the water maze suggests that these rats most probably had difficulty in retrieving spatial memory in both tasks. The fact that $\mathrm{mPFC}$ and ReRh rats subjected to MSCI infusions showed recall in the water-maze task but performance was weaker in ReRh rats indicates that neither mPFC nor ReRh contributes crucially to recent spatial memory retrieval. In several experiments, it has been shown that alterations of the mPFC affect the capability of shifting to the correct strategy when task contingencies or environmental conditions are modified (Ragozzino et al., 1999; Dias and Aggleton, 2000; Block et al., 2007; Jo et al., 2007; Oualian and Gisquet-Verrier, 2010). It is very possible that, after mPFC inactivation, rats simply were unable to properly manage this shift to

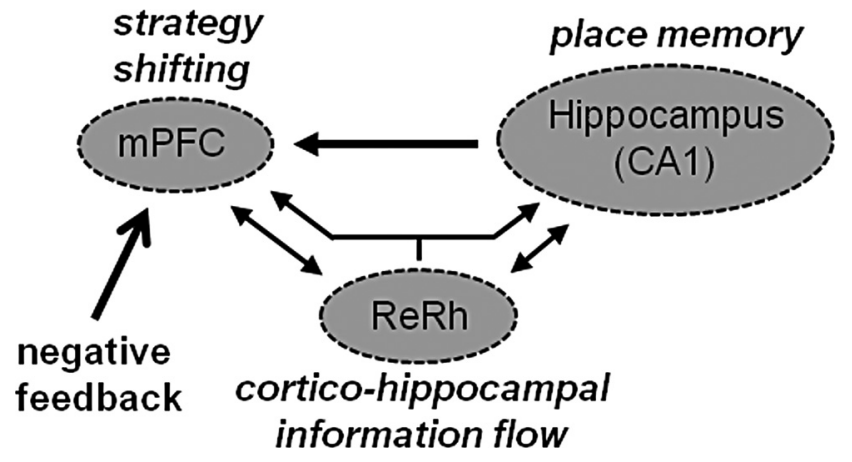

Figure 9. Hypothetical model of functional processes engaged in the probe trial of the double-H maze test. The hippocampus, the MPFC, and the ReRh of the ventral midline thalamus belong to a system characterized by a connectivity encompassing direct projections from the hippocampus to the $\mathrm{MPFC}$, reciprocal connections of the ReRh with the $\mathrm{mPFC}$ and with the hippocampus, and, in the Re, neurons having axon projecting collaterals in each of the cortical and hippocampal terminal fields; there are no described direct mPFC-hippocampal return projections (Hoover and Vertes, 2012). Our double-H maze data are compatible with a functional model in which negative feedback may trigger an $\mathrm{MPFC}$-dependent shift toward a hippocampus-dependent strategy engaging a place memory. The crucial functional relay in this cooperation could be the ReRh nuclei, which might serve as a hub relaying the information flow arising from the $\mathrm{mPFC}$ to the hippocampus. This hypothetical functional schema is compatible with the currently reported findings and provides a conceptual frame to further the investigations on the functional implications of the nuclei of the ventral midline thalamus.

a place memory-based behavior despite their intact memory for the training location of the platform. Why then the same effects after ReRh inactivation? An adapted behavior presupposes not only the mPFC-dependent shift to be operational but also the memory for place to be conjointly engaged, a process for which the hippocampus is important. In other words, most adapted behaviors require interactions between $\mathrm{mPFC}$ (for shifting) and dHip (for place memory retrieval). In all tasks requiring such interactions and in which the effects of ventral midline thalamus lesion or inactivation have been explored, deficits were found. Hembrook et al. (2012) showed impaired delayed nonmatching-to-position performance in operant chambers after ReRh inactivation, a task sensitive to either mPFC or hippocampal lesions. They also reported that permanent ReRh lesions altered win-shift performance in a radial maze, a task requiring coordinated activation of $\mathrm{mPFC}$ and hippocampus (Hembrook and Mair, 2011). Finally, we found that permanent ReRh lesions obliterated spatial memory persistence (Loureiro et al., 2012), for which cortico-hippocampal interactions are also crucial (Frankland and Bontempi, 2005). These findings together with the current ones are in agreement with neuroanatomical and electrophysiological investigations. First, ReRh neurons have, among other sites, dense overlapping terminal fields in region CA1 of the hippocampus and in the mPFC (Vertes et al., 2006). A recent study even found a subpopulation of Re neurons with axon collaterals in each terminal field (Hoover and Vertes, 2012). Because there are direct projections from the hippocampus to the $\mathrm{MPFC}$ but no direct return projection from the $\mathrm{MPFC}$ to the hippocampus (Thierry et al., 2000), the ReRh appears as a crucial relay in cortico-hippocampo-cortical information exchange (Fig. 9). Second, Di Prisco and Vertes (2006) reported that stimulation of the ReRh produced strong, monosynaptic activation of the PFC (Eleore et al., 2011). Dolleman-Van der Weel et al. (1997) showed previously that stimulation of the Re induced strong activity changes in the CA1 region of the hippocampus. Furthermore, Zhang et al. (2012) reported that systemic blockade of NMDA receptors by ketamine produced a coherent increase of activity in 
both the Re and hippocampus, and blockade of the Re by MSCI infusion reduced the ketamine-induced changes in the hippocampus. Despite these arguments and because not all corticohippocampal pathways have been disrupted by the ReRh inactivation in the current experiments (e.g., those passing through the rhinal cortex and others are still operational), an alternative interpretation of our data could be that the ventral midline thalamus is in fact crucial to $\mathrm{mPFC}$ functions and that the deficits found after mPFC or ReRh inactivation were in fact very similar in nature. Given the data recently reported by $\mathrm{Xu}$ and Südhof (2013) on the role of the Re nucleus in contextual fear memory generalization, the question of whether the deficits found in the double-H maze after ReRh inactivation could be explained by spatial memory generalization might be worth an investigation.

\section{Conclusions}

Altogether, our current data indicate that, in the double-H maze, a behavioral adaptation to a task contingency modification requiring a shift toward the use of a memory for location might operate in a distributed circuit encompassing the mPFC as the possible shift-triggering (or inappropriate strategy inhibiting) structure, the hippocampus as the substrate from which the place memory is retrieved, as well as the ventral midline thalamus, and therein the ReRh, either as a potential relay of this processing or as a crucial actor of mPFC functions (Fig. 9). Therefore, the ReRh could be an important subcortical contributor to behavioral flexibility. Beside their fundamental interest, these findings add to our understanding of the cognitive alterations characterizing diencephalic amnesia associated with stroke or Korsakoff's syndrome. They could also be of some interest regarding an interpretation of cognitive symptoms seen in schizophrenia, because the reunions nucleus was recently proposed as a possible link of a loop contributing to the psychotic break in schizophrenic patients (Lisman et al., 2010; Zhang et al., 2012). Finally, these results might also shed additional light on our understanding of neurological or psychiatric diseases characterized by symptoms of cognitive flexibility failures.

\section{References}

Aggleton JP, Brown MW (1999) Episodic memory, amnesia, and the hippocampal-anterior thalamic axis. Behav Brain Sci 22:425-444; discussion 444-489. Medline

Aggleton JP, Dumont JR, Warburton EC (2011) Unraveling the contributions of the diencephalon to recognition memory: a review. Learn Mem 18:384-400. CrossRef Medline

Allen TA, Narayanan NS, Kholodar-Smith DB, Zhao Y, Laubach M, Brown TH (2008) Imaging the spread of reversible brain inactivations using fluorescent muscimol. J Neurosci Methods 171:30-38. CrossRef Medline

Block AE, Dhanji H, Thompson-Tardif SF, Floresco SB (2007) Thalamicprefrontal cortical-ventral striatal circuitry mediates dissociable components of strategy set shifting. Cereb Cortex 17:1625-1636. CrossRef Medline

Blum S, Hebert AE, Dash PK (2006) A role for the prefrontal cortex in recall of recent and remote memories. Neuroreport 17:341-344. CrossRef Medline

Broadbent NJ, Squire LR, Clark RE (2006) Reversible hippocampal lesions disrupt water maze performance during both recent and remote memory tests. Learn Mem 13:187-191. CrossRef Medline

Carlesimo GA, Costa A, Serra L, Bozzali M, Fadda L, Caltagirone C (2011) Prospective memory in thalamic amnesia. Neuropsychologia 49:21992208. CrossRef Medline

Cassel R, Kelche C, Lecourtier L, Cassel JC (2012) The match/mismatch of visuo-spatial cues between acquisition and retrieval contexts influences the expression of response vs. place memory in rats. Behav Brain Res 230:333-342. CrossRef Medline
Churchwell JC, Kesner RP (2011) Hippocampal-prefrontal dynamics in spatial working memory: interactions and independent parallel processing. Behav Brain Res 225:389-395. CrossRef Medline

Di Prisco GV, Vertes RP (2006) Excitatory actions of the ventral midline (rhomboid/reuniens) on the medial prefrontal cortex in the rat. Synapse 60:45-55. CrossRef Medline

Dias R, Aggleton JP (2000) Effects of selective excitotoxic prefrontal lesions on acquisition of nonmatching- and matching-to-place in the T-maze in the rat: differential involvement of the prelimbic-infralimbic and anterior cingulate cortices in providing behavioral flexibility. Eur J Neurosci 12: 4457-4466. CrossRef Medline

Dolleman-Van der Weel MJ, Witter MP (1996) Projections from the nucleus reuniens thalami to the entorhinal cortex, hippocampal field CA1, and the subiculum in the rat arise from different populations of neurons. J Comp Neurol 364:637-650. CrossRef Medline

Dolleman-Van der Weel MJ, Lopes da Silva FH, Witter MP (1997) Nucleus reuniens thalami modulates activity in hippocampal field CA1 through excitatory and inhibitory mechanisms. J Neurosci 17:5640-5650. Medline

Dolleman-van der Weel MJ, Morris RG, Witter MP (2009) Neurotoxic lesions of the thalamic reuniens or mediodorsal nucleus in rats affect non-mnemonic aspects of watermaze learning. Brain Struct Funct 213:329-342. CrossRef Medline

Edeline JM, Hars B, Hennevin E, Cotillon N (2002) Muscimol diffusion after intracerebral microinjections: a reevaluation based on electrophysiological and autoradiographic quantifications. Neurobiol Learn Mem 78: 100-124. CrossRef Medline

Eleore L, López-Ramos JC, Guerra-Narbona R, Delgado-García JM (2011) Role of reuniens nucleus projections to the medial prefrontal cortex and to the hippocampal pyramidal CA1 area in associative learning. Plos One 6:e23538. CrossRef Medline

Floresco SB, Block AE, Tse MT (2008) Inactivation of the medial prefrontal cortex of the rat impairs strategy set-shifting, but not reversal learning, using a novel, automated procedure. Behav Brain Res 90:85-96. CrossRef Medline

Frankland PW, Bontempi B (2005) The organization of recent and remote memories. Nat Rev Neurosci 6:119-130. CrossRef Medline

Gold JJ, Squire LR (2006) The anatomy of amnesia: neurohistological analysis of three new cases. Learn Mem 13:699-710. CrossRef Medline

Hembrook JR, Mair RG (2011) Lesions of reuniens and rhomboid thalamic nuclei impair radial maze win-shift performance. Hippocampus 21:815826. CrossRef Medline

Hembrook JR, Onos KD, Mair RG (2012) inactivation of ventral midline thalamus produces selective spatial delayed conditional discrimination impairment in the rat. Hippocampus 22:853-860. CrossRef Medline

Hoover WB, Vertes RP (2012) Collateral projections from nucleus reuniens of thalamus to hippocampus and medial prefrontal cortex in the rat: a single and double retrograde fluorescent labeling study. Brain Struct Funct 217:191-209. CrossRef Medline

Jo YS, Park EH, Kim IH, Park SK, Kim H, Kim HT, Choi JS (2007) The medial prefrontal cortex is involved in spatial memory retrieval under partial-cue conditions. J Neurosci 27:13567-13578. CrossRef Medline

Kirch RD, Meyer PT, Geisler S, Braun F, Gehrig S, Langen KJ, von Hörsten S, Nikkhah G, Cassel JC, Döbrössy MD (2013) Early deficits in declarative and procedural memory dependent behavioral function in a transgenic rat model of Huntington's disease. Behav Brain Res 239:15-26. CrossRef Medline

Klur S, Muller C, Pereira de Vasconcelos A, Ballard T, Lopez J, Galani R, Certa U, Cassel JC (2009) Hippocampal-dependent spatial memory functions might be lateralized in rats: an approach combining gene expression profiling and reversible inactivation. Hippocampus 19:800-816. CrossRef Medline

Lecourtier L, Antal MC, Cosquer B, Schumacher A, Samama B, Angst MJ, Ferrandon A, Koning E, Cassel JC, Nehlig A (2012) Intact neurobehavioral development and dramatic impairments of procedural-like memory following neonatal ventral hippocampal lesion in rats. Neuroscience 207: 110-123. CrossRef Medline

Lisman JE, Pi HJ, Zhang Y, Otmakhova NA (2010) A thalamohippocampal-ventral tegmental area loop may produce the positive feedback that underlies the psychotic break in schizophrenia. Biol Psychiat 68:17-24. CrossRef Medline

Lopez J, Wolff M, Lecourtier L, Cosquer B, Bontempi B, Dalrymple-Alford J, 
Cassel JC (2009) The intralaminar thalamic nuclei contribute to remote spatial memory. J Neurosci 29:3302-3306. CrossRef Medline

Lopez J, Herbeaux K, Cosquer B, Engeln M, Muller C, Lazarus C, Kelche C, Bontempi B, Cassel JC, de Vasconcelos AP (2012) Context-dependent modulation of hippocampal and cortical recruitment during remote spatial memory retrieval. Hippocampus 22:827-841. CrossRef Medline

Loureiro M, Cholvin T, Lopez J, Merienne N, Latreche A, Cosquer B, Geiger K, Kelche C, Cassel JC, Pereira de Vasconcelos A (2012) The ventral midline thalamus (reuniens and rhomboid nuclei) contributes to the persistence of spatial memory in rats. J Neurosci 32:9947-9959. CrossRef Medline

Oualian C, Gisquet-Verrier P (2010) The differential involvement of the prelimbic and infralimbic cortices in response conflict affects behavioral flexibility in rats trained in a new automated strategy-switching task. Learn Mem 17:654-668. CrossRef Medline

Paxinos G, Watson C (1998) The rat brain in stereotaxic coordinates, Ed 4. San Diego: Academic.

Pol-Bodetto S, Jeltsch-David H, Lecourtier L, Rusnac N, Mam-Lam-Fook C, Cosquer B, Geiger K, Cassel JC (2011) The double-H maze test, a novel, simple, water-escape memory task: acquisition, recall of recent and remote memory, and effects of systemic muscarinic or NMDA receptor blockade during training. Behav Brain Res 218:138-151. CrossRef Medline

Ragozzino ME, Detrick S, Kesner RP (1999) Involvement of the prelimbicinfralimbic areas of the rodent prefrontal cortex in behavioral flexibility for place and response learning. J Neurosci 19:4585-4594. Medline

Ragozzino ME, Kim J, Hassert D, Minniti N, Kiang C (2003) The contribution of the rat prelimbic-infralimbic areas to different forms of task switching. Behav Neurosci 117:1054-1065. CrossRef Medline

Schumacher A, de Vasconcelos AP, Lecourtier L, Moser A, Cassel JC (2011) Electrical high frequency stimulation in the dorsal striatum: effects on response learning and GABA levels in rats. Behav Brain Res 222:368-374. Medline

Stratford TR, Wirtshafter D (2013) Injections of muscimol into the para- ventricular thalamic nucleus, but not mediodorsal thalamic nuclei, induce feeding in rats. Brain Res 1490:128-133. CrossRef Medline

Su HS, Bentivoglio M (1990) Thalamic midline cell populations projecting to the nucleus accumbens, amygdala, and hippocampus in the rat. J Comp Neurol 297:582-593. CrossRef Medline

Teixeira CM, Pomedli SR, Maei HR, Kee N, Frankland PW (2006) Involvement of the anterior cingulate cortex in the expression of remote spatial memory. J Neurosci 26:7555-7564. CrossRef Medline

Thierry AM, Gioanni Y, Dégénétais E, Glowinski J (2000) Hippocampoprefrontal cortex pathway: anatomical and electrophysiological characteristics. Hippocampus 1:411-419. CrossRef Medline

Van der Werf YD, Jolles J, Witter MP, Uylings HB (2003) Contributions of thalamic nuclei to declarative memory functioning. Cortex 39: 1047-1062. CrossRef Medline

van Duuren E, van der Plasse G, van der Blom R, Joosten RN, Mulder AB, Pennartz CM, Feenstra MG (2007) Pharmacological manipulation of neuronal ensemble activity by reverse microdialysis in freely moving rats: a comparative study of the effects of tetrodotoxin, lidocaine, and muscimol. J Pharmacol Exp Ther 323:61-69. CrossRef Medline

Vertes RP (2006) Interactions among the medial prefrontal cortex, hippocampus and midline thalamus in emotional and cognitive processing in the rat. Neuroscience 142:1-20. CrossRef Medline

Vertes RP, Hoover WB, Do Valle AC, Sherman A, Rodriguez JJ (2006) Efferent projections of reuniens and rhomboid nuclei of the thalamus in the Rat. J Comp Neurol 499:768-796. CrossRef Medline

Vertes RP, Hoover WB, Szigeti-Buck K, Leranth C (2007) Nucleus reuniens of the midline thalamus: link between the medial prefrontal cortex and the hippocampus. Brain Res Bull 71:601-609. CrossRef Medline

Xu W, Südhof TC (2013) A neural circuit for memory specificity and generalization. Science 339:1290-1295. CrossRef Medline

Zhang Y, Yoshida T, Katz DB, Lisman JE (2012) NMDAR antagonist action in thalamus imposes delta oscillations on the hippocampus. J Neurophysiol 107:3181-3189. CrossRef Medline 